\title{
Pre Clinical- Basic Sciences Teaching Curriculum Of A Medical School in A Developing Country -Are We Doing It Right?
}

\author{
Kushani Rasangika Atukorala ${ }^{1}$, Piyusha Atapattu ${ }^{2}$
}

\begin{abstract}
Background: Preclinical teaching of basic sciences provides the basis for the development of clinical reasoning skills and the ability to make management decisions. However, many senior undergraduates, pre-interns and doctors indicate that basic sciences knowledge is poorly recalled and has little relevance to their clinical practice. Objectives: To explore the perceptions of medical students in their clinical years, and pre-interns about the basic sciences courses taught to them in the preclinical years, and to assess how each group rates the applicability of these courses to current clinical training. Methods: A descriptive cross-sectional study was conducted among 118 pre-interns and 146 undergraduate medical students using a self-administered questionnaire, regarding their perceptions on preclinical basic sciences teaching. For statistical analysis chi square test was applied. Results: More than $75 \%$ both pre-interns and undergraduates agreed that preclinical teaching was useful and relevant for future clinical work. 50-75\% frequently revisited preclinical subjects despite unapparent clinical significance. $55 \%$ couldn't remember most of preclinical content. Physiology was the most retained (76\%) and most clinically relevant subject ( $80 \%)$. Majority of $(>60 \%)$ both the groups suggested more teaching time and $>75 \%$ suggested concurrent clinical exposure for preclinical teaching. Undergraduates and preinterns differed in that $56 \%$ of undergraduates and $37 \%$ of pre-interns had studied pre clinical subjects just to pass examinations $(\mathrm{p}<0.01)$.Conclusions: Majority of undergraduates and pre-interns felt that preclinical teaching is interesting and relevant for future clinical practice, though recall and clinical significance were suboptimal. Revising teaching methods with interdisciplinary integration, early clinical exposure showing relevance of basic sciences and allocating more teaching time utilizing clinicians should be considered.
\end{abstract}

Key words- Pre Clinical teaching, Basic sciences, Physiology, Undergraduates

Bangladesh Soc Physiol. 2014, December; 9(2): 98-104 For Authors Affiliation, see end of text.

http://www.banglajol.info/index.php/JBSP

\section{Introduction}

I $\mathrm{t}$ is generally accepted that medical students should learn basic sciences which enables medicine to be rational ${ }^{1}$. Three primary roles for the basic sciences in medical education are identified: to provide the basis for the development of clinical reasoning

Received September 2014; Accepted November 2014 skills and the ability to make management decisions ,to support critical analysis of medical and surgical interventions and analysis of processes to improve healthcare ${ }^{2}$. Studies have demonstrated that basic science instruction greatly enhanced the learning of novel related content, ${ }^{3}$ thus a strong basic science background provides the conceptual framework 
against which to evaluate new scientific and medical findings and makes it easier for physicians to adapt to changes in knowledge and practice evidence based medicine

However, traditional programs of teaching basic sciences have failed to provide better outcomes of learning as lack of integration of basic sciences with clinical sciences have often been perceived by students as lack of relevance of basic science to clinical medicine ${ }^{4,5}$

For this reason the current curriculum at Faculty of Medicine, University of Colombo pioneered and devotes the first 3-4 years to basic and applied sciences teaching, where basic sciences are taught in both the Basic Sciences Stream and the Applied Sciences Stream. Emphasis is placed in the early years on how the knowledge or skills acquired at the Basic Sciences Stream will be used in courses taught at a later stage. From the second year onwards in the Applied Sciences Stream, basic sciences are integrated to the clinical sciences, to facilitate development of knowledge that is relevant and meaningful to clinical practice which is deep and retrievable and results in producing lifelong learners.

Even after many schools have made substantial changes in their curriculum to promote integration of basic and clinical sciences, students still seem to have difficulty in retaining basic science information ${ }^{6-9}$. A similar situation is seen in the local context. Many senior undergraduate students have indicated informally that they did not remember much from their basic science subjects and that the content of those courses did not seem relevant to their later clinical studies or clinical work. Surely if students are not remembering what they have been taught then the effort has been wasted; if students cannot make use of the knowledge they have been taught, if that knowledge becomes inert and inaccessible, then why teach it in the first place? ${ }^{10-12}$.

It is possible that student achievements and future practice are affected by their attitudes towards basic sciences courses. Therefore it is worth exploring the perceptions of medical students in their clinical years, and pre-interns who are just about to start their clinical career, about the basic sciences courses taught to them in the preclinical years, and to assess how each group rates the applicability of these courses to current clinical training.

\section{Methods}

A descriptive cross-sectional study was conducted in faculty of medicine, University of Colombo, from 2011 to 2012 among 118 pre-interns and 146 undergraduates in their fourth year regarding their perceptions on preclinical basic sciences teaching, using a self-administered questionnaire. Ethical approval was obtained from the Ethics Review committee of the Faculty of Medicine, University of Colombo. The participants were selected randomly and verbal consent was obtained prior to their participation in the study.

Data were collected using an anonymous, predesigned, self-administered questionnaire. Pretesting was conducted using a sample of 20 students, who were asked to comment on the questionnaire's content, consistency, clarity and appearance. Modifications were adopted as recommended. Undergraduate questionnaires were administered when the whole batch was gathered for clinical lectures at the common lecture theatre of faculty of medicine of University of Colombo. Questionnaires were administered at the end of the lecture. Pre-intern questionnaires were distributed when they gathered for a common lecture/workshop (about internship) before starting the internship at the lecture theatre.

The questionnaire collected information on students' academic year and their perceptions of the following characteristics of their basic sciences courses (anatomy, pathology, biochemistry, microbiology and physiology, pharmacology): organization of the content, recall 
of information, and applicability to clinical practice.

All opinions were rated using the 5-point Likert scale, which ranged from "strongly disagree" to "strongly agree." Although all opinion levels were initially analyzed, percentages of those who responded as either "strongly agree" or "agree" were merged into a single category, and tables were prepared so as to present only positive responses. Data were arranged as numbers and percentages. All responses were stratified separately as undergraduate/pre intern. Chisquare tests were performed on categorical variables, and significance was defined as $P<0.05$.

\section{Results}

Of the 264 participants (56\% males), $118(44.7 \%$ ) were fourth-year students and $146(55.3 \%)$ were pre-intern medical graduates.123/264 (46.6\%) of the participants had obtained a Class at the final examination of the Basic Sciences Stream.
Retention of basic sciences Knowledge Overall, majority of participants 151/264 (57\%) could not remember most of the preclinical content learned (Table I). Majority 201/264 (76\%) indicated that most of the basic sciences knowledge retained was in physiology. (Figure 1) The subjects in which the least knowledge was retained by the majority were anatomy 166/264(63\%) and biochemistry 74/264 (28\%). There was no statistically significant difference in retaining preclinical knowledge in the two groups $(\mathrm{p}>0.05)$ as majority of participants $75 / 146(51 \%) / 72 / 118(61 \%)$ in both groups stated that they cannot remember most of what was taught, although one would expect the undergraduates to remember the content better, as they may require frequent recall of basic sciences during clinical studies and as content learnt was fresh in their minds. Obtaining a class at the final examination of the Basic Sciences Stream did not have a significant correlation with retention of preclinical knowledge as there was no significant difference in retaining preclinical

Table I: Participant response regarding basic sciences teaching

\begin{tabular}{|c|c|c|c|c|c|}
\hline & \multicolumn{2}{|c|}{ Undergraduates $(n=146)$} & \multicolumn{2}{|c|}{ Pre-interns $(n=118)$} \\
\hline \multicolumn{2}{|c|}{ Questions } & \multirow{2}{*}{$\frac{\text { Yes }}{124(84.9 \%)}$} & \multirow{2}{*}{$\frac{\text { No }}{22(15 \%)}$} & \multirow{2}{*}{$\frac{\text { Yes }}{94(79.6 \%)}$} & \multirow{2}{*}{$\frac{\text { No }}{24(20.3 \%)}$} \\
\hline 1. & $\begin{array}{l}\text { The pre-clinical teaching was important/useful } \\
\text { in doing your clinical work }\end{array}$ & & & & \\
\hline 2. & $\begin{array}{l}\text { When you were training in the clinical setting } \\
\text { you had to frequently revisit these subjects. }\end{array}$ & $102(69.9 \%)$ & $44(30.13 \%)$ & $76(64.4 \%)$ & $42(35.6 \%)$ \\
\hline 3. & You found the subjects interesting to learn & $109(74.6 \%)$ & $37(25.3 \%)$ & $78(66 \%)$ & $40(33.9 \%)$ \\
\hline 4. & $\begin{array}{l}\text { Pre clinical curriculum is overloaded with lot } \\
\text { of unimportant content }\end{array}$ & $128(87.6 \%)$ & $18(12.3 \%)$ & $101(85.6 \%)$ & $18(15.2 \%)$ \\
\hline 5. & $\begin{array}{l}\text { You remember the content of the subject very } \\
\text { well even now }\end{array}$ & $71(48.6 \%)$ & $75(51.2 \%)$ & $49(41.5 \%)$ & $72(61 \%)$ \\
\hline 6. & You studied just to pass the subject & $82(56.2 \%)$ & $64(43.8 \%)$ & $44(30.13 \%)$ & $74(62.7 \%)$ \\
\hline 7. & $\begin{array}{l}\text { Clinical work should be introduced to } \\
\text { pre clinical teaching. }\end{array}$ & $123(84.2 \%)$ & $23(75.7 \%)$ & $93(78.8 \%)$ & $2521.2 \%)$ \\
\hline 8. & The content taught, lacked clinical relevance. & $97(66.4 \%)$ & $49(33.6 \%)$ & $70(47.9 \%)$ & $48(40.6 \%)$ \\
\hline 9. & $\begin{array}{l}\text { Content taught provided relevant preparation } \\
\text { for clinical rotations/clerkships. }\end{array}$ & $61(41.7 \%)$ & $85(58.2 \%)$ & $42(35.6 \%)$ & $66(55.9 \%)$ \\
\hline 10. & $\begin{array}{l}\text { Pre clinical teaching is best done by } \\
\text { academicsrather than clinicians. }\end{array}$ & $97(66.4 \%)$ & $49(33.6 \%)$ & $54(45.7 \%)$ & $64(54.2 \%)$ \\
\hline
\end{tabular}




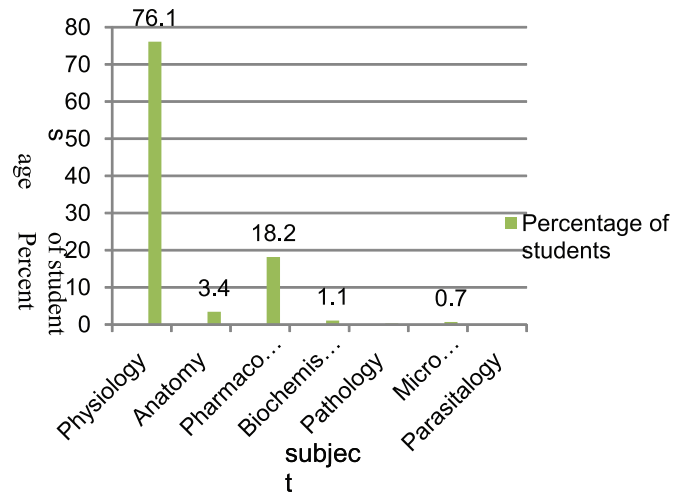

Figure 1: Most retained subject by the participants

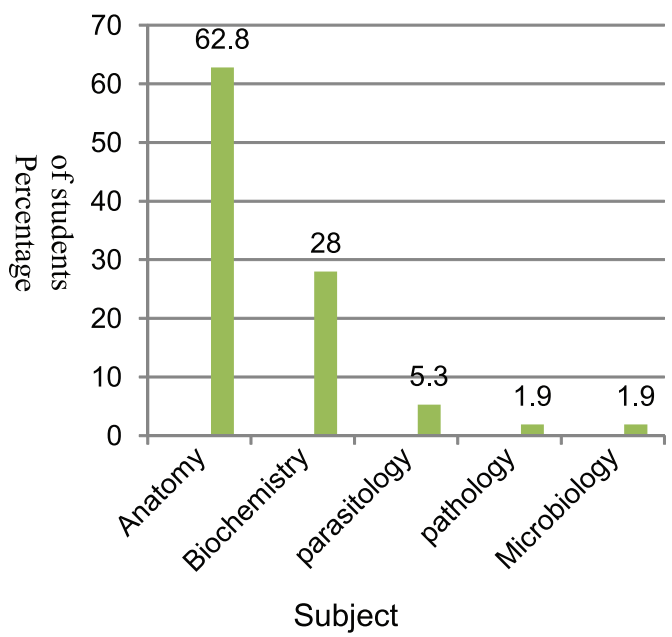

Figure 2: Least retained subject by the participants

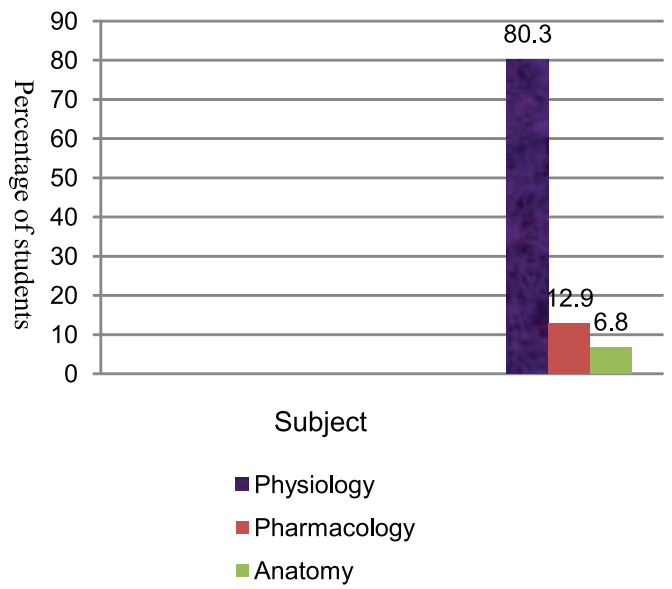

Figure 3: Most clinically relevant subject knowledge in the group with classes and the group without classes. $\mathrm{p}>0.05$.

Clinical applicability

When it came to how they relate the importance of the preclinical basic science teaching on their clinical rotations, majority 218/264 (82.5\%) thought that it is helpful, relevant and important. However, majority 150/264 (56.8\%) from both groups had said that basic sciences teaching was not helpful in preparing them for clinical clerkships/clinical rotations. At the same time the majority 167/264 (63.2\%) stated that overall most of the basic sciences teaching lacked clinical significance and that they are not satisfied with basic sciences teaching. Physiology was perceived as the subject with the highest applicability to clinical practice by $212 / 264$ (80\%) followed by pharmacology 44/264 (16.6\%) by all participants.

Majority of had to revisit the subjects during the clinical years. 194/264 (73.5\%) of all participants have indicated that out of the preclinical subjects physiology should have the most teaching time. 216/264 (81.8\%) thought that clinical work should be introduced in to pre clinical teaching with concurrent clinical exposure.

Undergraduates and pre-interns differed in that $82 / 146(56 \%)$ of undergraduates and 44/118 (37\%) of pre-interns had studied just to pass examinations $(\mathrm{p}<0.01)$. Though $97 / 146$ (66.4\%)undergraduates now in training believed that pre clinical teaching in general should be done by academics, the majority of pre-interns [64/118 (54.3\%)]thought that clinicians should do it $(\mathrm{p}<0.01)$

\section{Discussion}

This study has revealed the stark realities of the status of the current undergraduate basic sciences program as perceived by the students. Majority of participants stated that basic sciences were interesting, important in their clinical work and more time should be allocated for preclinical 
teaching. The majority of students frequently revisited the basic sciences during clinical years. However, the recall of basic sciences was suboptimal and decreased with time. The majority stated that most of the basic sciences teaching lacked clinical significance and they were not satisfied with it.

Both undergraduates and pre-interns were positive regarding the basic sciences learned, similar to that observed in some other settings 13 , but not regarding the basic sciences 'teaching' they experienced. Therefore it appears that regardless of the time allocated for preclinical teaching, the way that it is taught has to be changed. Integrating clinical teaching with the basic sciences during preclinical years will provide continuity and a seamless transition from preclinical to clinical disciplines. Early clinical exposure could enhance comprehending the clinical applications of basic sciences, promoting deep learning which will enhance the retention of basic sciences. This becomes evident from our study findings, as both the groups thought that clinical work should be introduced in to preclinical teaching from the beginning. Similarly, in another study $70 \%$ were dissatisfied with the organization of the basic sciences content, and only $23 \%$ believed that the content taught in this phase prepared them for clinical clerkship ${ }^{14}$. When asked to give their opinion about the relevance of the specific basic science courses in preparation for clinical work, between 59.4\% and $72.5 \%$ of the respondents indicated that the following courses - biochemistry and genetics had little if any relevance to their work in the clinical setting. Those ranked highest among basic science courses in being preparatory for clinical experience were physiology, general pathology and anatomy $(61.2 \%, 47 \%$, and $44.3 \%$ respectively) ${ }^{14}$. In another study about $94 \%$ of the students agreed that studying physiology is relevant for studying other subjects and that it is relevant to their future professional career ${ }^{15}$. Our study shows similar findings where physiology is favored by both undergraduates and preinterns as the most clinically relevant as well as the best recalled subject. The preferential recall and perceived clinical applicability of physiology could be due to the ease with which the connections between physiology and clinical situations could be demonstrated during lectures and practicals, which is not so apparent in subjects like biochemistry or genetics.

Though the majority of undergraduates believed that preclinical teaching should be done by academics, the majority of pre-interns preferred clinicians as basic sciences teachers. The difference in opinion could be attributed to the fact that the pre-interns with more clinical exposure would have felt that the basic sciences knowledge required by the clinicians for clinical practice is different to what is stressed at the preclinical stage. Once a student completed the preclinical years, he or she would soon learn that clinicians do not need much basic science knowledge to solve many clinical problems - the clinician has seen it before and has learned the appropriate response. This could lead to a "dissonance" that many students encounter in the clinical years.

Although many medical schools around the globe (including much of Asia) have made substantial changes in their curriculum to promote integration of basic and clinical sciences, medical students in the clinical years still seem to have poor retention of and, therefore, lack the ability to apply basic science knowledge, concepts and principles acquired in the preclinical years. The results in our study have been no different. On the whole, in our study, retention of basic science knowledge was suboptimal. It is in keeping with the marked decline in retention of basic medical sciences observed among the undergraduates, which is documented in the literature ${ }^{14,16-18}$. Though a comprehensive understanding of the basic sciences is essential for the diagnosis and management in clinical practice, the in-depth knowledge of basic 
sciences taught in the early years is likely to be lost with time, especially when not frequently recalled for clinical practice. Multiple studies have further confirmed that students' retention of noncontextualized basic science factual information is poor and basic science education, without significant clinical context, has resulted in shortbut not long-term retention of knowledge ${ }^{16,19-}$ 20. Physiology which was rated as the most clinically relevant of all pre clinical subjects was found to be the mostly retained subject. This finding confirms that perceived relevance of a subject matter fosters retention of knowledge as seen in other studies ${ }^{21}$. Similarly in one study, approximately, $65 \%$ of the respondents were able to recall the knowledge of anatomy and physiology while biochemistry was retained by $40 \%$. Students identified anatomy as the subject which overloaded syllabus (75.4\%) and also with maximum clinical application with $50.1 \%$ of them considering it the most important basic sciences subject $^{13}$.

Majority of undergraduates stated that they studied just to pass the subject though it was not so with the pre-interns. This may show an attitudinal change in the current undergraduates towards retaining preclinical knowledge in comparison to the pre-interns. The reason for this attitude has to be explored as there lies a great danger, as students may not fully utilize the opportunity to learn the foundations of medicine. One reason for poor retention also could be that students may have mainly concentrated on ways to gain marks, rather than focusing on how the information could be applied in clinical settings. Though the higher achievers with a Class at the Introductory Basic Sciences Stream are expected to have better initial knowledge and therefore retention and recall of basic sciences, there was no significant difference in retaining preclinical knowledge between participants with and without classes. It is believed that for many students, basic science has been reduced to a rite of passage, characterized by binging and then purging after completing the basic science-centric component of the licensure examination (USMLE Step 1) ${ }^{22}$. This is a pathetic situation, because, physicians must be constantly learning, and that learning is much easier if they have a firm background of the physiological, biochemical, and anatomical principles involved, even if they quickly forget minute specific details.

If students resort to rote learning just to score high marks at the examinations, as stated by the majority of undergraduates in our study, the main purpose of basic sciences teaching is lost. The causes of this behavior should be studied extensively to see whether what prompts students to just memorize facts eg. overloaded curriculum, lack of clinical relevance in the content etc.... There is strong consensus that appropriate integration in the teaching-learning of the basic medical sciences with clinical medicine will achieve the desired outcome. ${ }^{22-23}$

\section{Conclusions}

Majority of undergraduates and pre-interns felt that preclinical teaching is interesting and relevant for future clinical practice, though recall and clinical significance of what was taught were suboptimal. Revising teaching methods with interdisciplinary integration, early clinical exposure showing relevance of basic sciences and allocating more teaching time utilizing clinicians should be considered.

In this context then, we need to seriously consider designing new and innovative curriculum structures which will ensure not only the clinical relevance, but also result in better understanding and student retention of basic science knowledge in the clinical years. How best can we optimize integration of the basic sciences with clinical medicine? Basic science curriculum needs to focus less on recall of facts and more on application of basic science principles to solve problems. Curricular models in which students learn scientific fundamentals in health-care 
contexts which will help students comprehend this knowledge and be more readily able to transfer and apply it to clinical decisions, should be developed as it will reinforce its perceived relevance to patient care positively.

\section{Acknowledgements}

Authors would like to thank the undergraduate and pre intern students who participated in the study

\section{Authors affiliations}

1. *Kushani Rasangika Atukorala, Lecturer , Department of Physiology, Faculty of Medical sciences, University of Sri Jayawardenapura, Sri Lanka. +94772321132, Email: kushaniatukorala (a)yahoo.com

2. Piyusha Atapattu, Senior Lecturer, Department of Physiology, Faculty of Medicine, University of Colombo, Sri Lanka.

\section{References}

1. Campbell E J M. Basic science, science and medical education. The Lancet. 1976;307(7951):134-136.

2. Grande J P. Training physicians for the twentyfirst century: role of the basic sciences. Medical teacher. 2009; 31 (9): 802-806.

3. LamisKaddam, Mustafa Khidir Mustafa Elnimeiri Students' perceived value of physiology course activities in a Sudanese medical faculty. Adv Physiol Educ 2012; 36: 298-301.

4. Khalid BA. The current status of medical education in the Gulf Cooperation Council countries. Ann Saudi Med 2008;28: 83-88.

5. Cooke M, Irby DM, Sullivan W, Ludmerer KM. American medical education 100 years after the Flexner report. N Engl J Med 2006; 355: 13391344.

6. LuAnn W, Stevens CM, Sally K. No Content Without Context: Integrating Basic, Clinical, and Social Sciences in a Pre-clerkship Curriculum. Med Teacher. 2009; 31: 812-821

7. Grande JP. Training of Physicians for the Twentyfirst Century: Role of the Basic Sciences. Med Teacher. 2009; 31: 802-806.

8. Ling Y, Swanson DB, Holzman K, Bucak S. Retention of Basic Science Information by Senior Medical Students. Acad Med. 2008; 83 (Suppl 10): S82-S85.

9. Spencer AL, Teresa B, Levine AS. Back to the Basic Sciences: An Innovative Approach to
Teaching Senior Medical Students How Best To Integrate Basic Science and Clinical Medicine. Acad Med. 2008; 83: 662-

10. Cate O, Snell L, Mann K, Vermunt J. Orienting teaching toward the learning process. Acad Med. 2004; 79:219-228.

11. Cox K. Knowledge which cannot be used is useless. Med Teacher. 1987;9:145-54.

12. Conway MA, Cohen G, Stanhope N. Very longterm memory for knowledge acquired at school and university. App Cognitive Psych. 1992;6: 467-82.

13. Jalili M, Mirzazadeh A, Azarpira A. Survey of Medical Students' Perceptions of the Quality of their Medical Education upon Graduation Ann Acad Med Singapore 2008;37:1012-18

14. Custers EJ, Ten Cate OT. Very long-term retention of basic science knowledge in doctors after graduation Med Educ. 2011;45(4):422-30.

15. Gupta S, Gupta A, Verma M, Kaur H, Kaur A, Singh $\mathrm{K}$. The attitudes and perceptions of medical students towards basic science subjects during their clinical years: A cross-sectional survey Intl J Appl Bas Medl Res2014; 4(1):16

16. D'Eon MF. Knowledge loss of medical students on first year basic science courses at the University of Saskatchewan. BMC Med Educ 2006; 6:5.

17. Swanson DB, Case SM, Luecht RM, Dillon GF. Retention of basic science information by fourthyear medical students. Acad Med. 1996 ;71(10 Suppl):S80-2.

18. Custers EJFM: Long-term retention of basic science knowledge: a review study. Adv Health Sci Educ Theory Pract. 2010; 15(1):109-128.

19. Ellis JS, Semb GB, Cole B. Very long-term memory for information taught in school. Contemp Educ Psych. 1998;23:419-33.

20. Watt ME. Retention of preclinical knowledge by clinical students. Med Educ. 1987 ;21(2):119-24.

21. Malau-Aduli BS, Lee AYS, Cooling N, Catchpole M, Jose $M$ and Turner R. Retention of knowledge and perceived relevance of basic sciences in an integrated case-based learning $(\mathrm{CBL})$ curriculum BMC Med Educ. 2013; 13:139

22. Fincher RE, Wallach PM, and Richardson WS. Basic Science Right, Not Basic Science Lite: Medical Education at a Crossroad. J Gen Intern Med. Nov 2009; 24(11): 1255-1258.

23. Gwee MCE, Samarasekera D \& Chay-Hoo T. Role of Basic Sciences in 21st Century Medical Education: An Asian Perspective Med Sci Educ 2010; 20(3) : 